\title{
Advanced Technique for Improved Mobile Multimedia Communication Services
}

\author{
U. Ukommi, M. Uko and U. Ekpe \\ uukommi@yahoo.com
}

\begin{abstract}
The proliferation of smartphones is associated with the development of multimedia applications. Mobile multimedia applications involve audio, data, speech, image, video processing and distribution of over mobile platform. However, compressed media packets are vulnerable to channel errors, thus making it difficult to sustain good perceived video quality performance within limited resources. In this research work, the weight length and impact of different video packets are analyzed. Based on the analysis an advanced technique to enhance mobile multimedia communication services is proposed. The technique involves cross-layer optimization framework, utilizing Network Abstraction Layer Units Length and flexibility of IP-based mobile network in exchanging network information for error protection of sensitive media packets. The simulation results carried out with compatible multiple media streams of different priority levels and Network Abstraction Layer Units Length show overall significant improvement in the received media services.
\end{abstract}

Keywords: Audio, communications, data, multimedia, network, quality enhancement, speech, video.

\section{Introduction}

The demand for mobile multimedia services is gradually increasing. The fear is that network level architecture and the air interface do not have sufficient capacity and flexibility to deliver real-time multimedia services at an acceptable quality of service level. Furthermore, the network operators and multimedia users are in need of optimized applications for distribution and consumption of such services including, mobile video services, emergency services for remote consultation, scene of crime work, virtual universities for remote learning, the security industry for telesurveillance, video telephony, business video conferencing, healthcare experts for remote diagnosis and monitoring. In these multimedia applications, video plays significant role. However, these multimedia services exert pressure on the limited mobile network resources due resource constraints and greater demand of improved multimedia services. Moreover, compressed media stream is susceptible to channel distortion due to certain factors including fading, interference, pathloss. These factors affect the performance of these applications. Media encoding algorithm supports error-resilient features such as data partitioning, intra update, slice interleaving for robustness of media stream over error prone channels. However, it is clear that source coding is no longer simply an issue of optimizing ratedistortion characteristics is not enough to combat the impact of channel distortions on received video quality, hence requires advanced protection technique to mitigate impact of channel errors and improve quality performance of received multimedia applications. Traditionally, channel errors can be controlled by existing technologies such as Automatic Re-transmission on Request approach where the corrupted video packets are retransmitted in response to receiver request. However, Automatic 
Re-transmission on Request incurred delays in process of retransmission of loss video packets. Hence, Automatic Re-transmission on Request is not suitable for delay sensitive video applications such as live football match playout and car racing videos. Channel coding such as Forward Error Correction maybe employed in video communication system to enhance the reliability of transmitted video streams over error prone channel. In Forward Error Correction, the additional video packets (redundancy) for protection incur more bandwidth requirement and delays. Advancement in mobile communication system has made it possible to exploit adaptive modulation scheme in improving the quality of video transmission over error prone channel. Several applications of adaptive modulation scheme are found in the literature [2] [3] [4], where the modulation parameters are adapted based on the channel conditions. In addition to the review of existing multimedia distribution technologies, improving the quality performance of mobile multimedia communication services based on the systematic adaptation of media packets is presented in this paper.

\section{The Proposed Technique}

The proposed advanced technique for improved multimedia communication services is discussed in this section. The aim of is to enhance the quality performance of multimedia services over mobile channel over constrained mobile network resources. In the proposed technique, the adaptation of media packets is based on the sensitivity of the media content to channel errors. The technique adopts cross-layer optimization framework which utilizes Network Abstraction Layer Units Length and flexibility of IP-based mobile network in exchanging network information for error protection of sensitive media packets. The media packets with significant amount of motion are highly prioritized compared to media contents with relative low amount of motion. In the proposed technique, the Network Abstraction Layer Unit (NALU) [1] of media streams with high motion characterization is prioritized and NALU adapted based on the intensity of the motion in the content. The NALU of media streams of low motion characterization are equally adapted differently. However, in order to avoid unbearable transmission overheads the NALU of media streams of highly prioritized packets are made relatively smaller compared to the NALU of media streams that is less sensitive to channel errors.

\section{System Design}

The system design of the proposed technique is presented in Figure 1. The system design consists of source coding, transmission and receiving chains. The source coding includes capturing of scene using video camera, filtering and encoding process. The source encoding involves removal of redundancies using algorithm. The transmission section involves channel coding and the receiving section consists of decoder and display unit. Figure 1 presents the proposed system architecture.

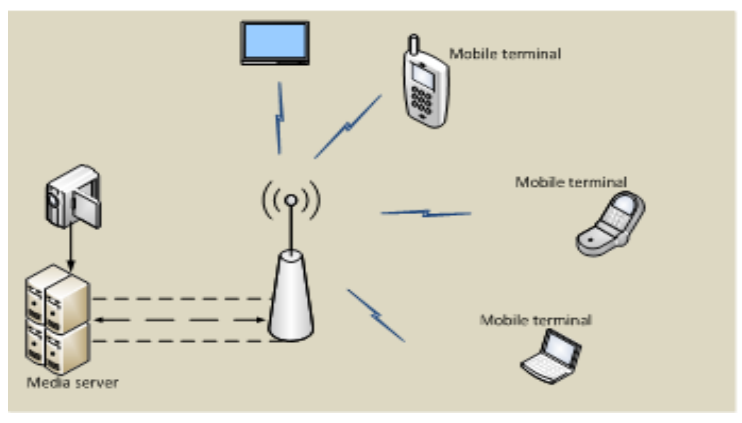

Figure 1: Proposed System 
Mobile multimedia applications such as video involves capturing of natural scene by video camera, encoding by media compression algorithms and distribution of the compressed media streams over a wireless channel. The encoding block performs media compression function by exploiting redundancies in video sequence and application of various algorithms to enhance robustness of the media streams. In the proposed technique, the transmission process significantly depends on the media packets sensitivity to channel errors, resource constraints and channel characteristics. Mobile multimedia communication is more challenging due to the limited bandwidth availability and high bit error rates capable of causing quality degradation on the received media performance. The transmitted media streams are processed at the receiver. The reconstructed media stream is processed and displayed on the receiving device. More details on multimedia communication including digital media compression, signal processing and decoding are discussed in the literature [5][6][7]. The model for estimating the sensitivity of media stream in terms of motion characterization is discussed in the literature [8]. However, the spatial and temporal resolutions of the video sequence and the number of frames in the test media stream are also taken into consideration in the simulation process.

\section{Experimental System Configuration}

The experimental process to measure efficiency of the proposed technique is discussed in this section. The experimental system configuration and simulation are performed to evaluate the proposed technique. In the system configuration, the content dynamic characterization is analyzed using optical flow algorithm of Lucas and Kanade [9]. Two standard test media sequences characterized with high and low motion characterizations are analyzed using the algorithm. The source coding is modeled using H.264/AVC reference software [10]. Simulated wireless channel model is used in the experimental work [11]. In the experiment, the media server stores different media contents of diverse motion characterization. The media encoder algorithm performs encoding and systematic packetization of NALU for improved transportation of media streams. The performance of the proposed technique is tested with two standard test media sequences: Football and Akiyo test media sequences, representing different types of media content services. The media source coding parameter setting include: Group of Picture GOP size of 8, frame rate of $30 \mathrm{fps}$. Common Intermediate Format. Each test media sequence has a total number of 900 frames. The received media streams are processed using H.264/AVC reference software. The channel performance is carried out with presimulated error patterns composed of traces of different Signal-to-Noise Ratio for different modulation schemes. The data slot error patterns are obtained by comparing the data bits within original data slot to the transmitted data slot. If there is any bit error within the data slot, it is then declared as an error. More details on path loss, fading and wireless network channel are available in the literature [13] [14].

The performance of the proposed scheme is measured using Peak-Signal-to-Noise-Ratio model. Peak Signal-to-Noise Ratio (PSNR) measures video quality by correlating the maximum possible value of the luminance and the mean squared error (MSE). The overall media quality performance is obtained by averaging the PSNR values throughout the video sequence. Higher PSNR values indicate better quality. Although, PSNR is not the most reliable metric of video quality assessment, it is employed in the research due to its less complexity, ease in calculation and widely usage for video quality assessment.

\section{Results and Discussions}

The quality performance of the proposed technique was tested with two standard media sequences in Common Intermediate Format. The tested media sequences include standard Football, and Akiyo test sequences. In the experiment, pre-encoded media streams are transmitted to the mobile terminal 
through the wireless simulator. The simulations were repeated 15 times to obtain stable results. The results are obtained by averaging the PSNR video quality performance values. Figure 2 presents the quality performance carried out with soccer test media sequence in terms of PSNR(dB).

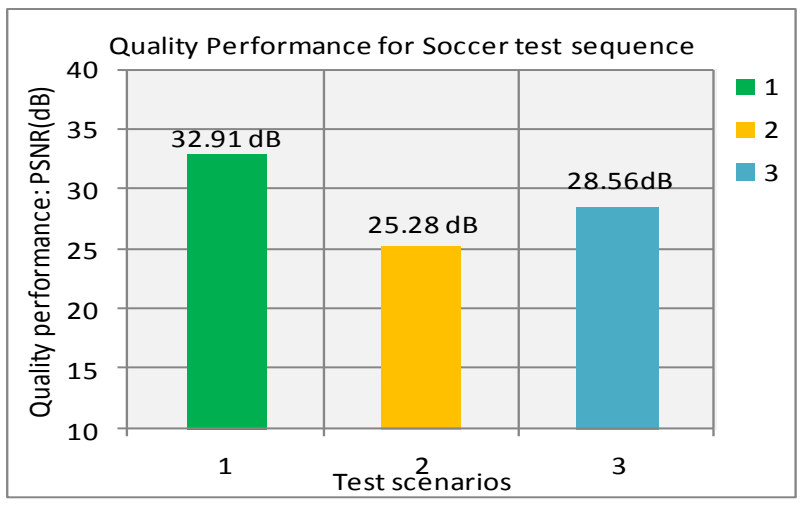

Figure 2: Quality Performance for Soccer test sequence.

Figure 2 presents the test results of the proposed technique performed carried out with soccer test media sequence, under three different scenarios. As shown in Figure 2, the test scenario 1 was performed under error free channel. This was necessary in order to verify the efficiency of the system at various test conditions. It is shown that the quality performance in test scenario 1 recorded the best performance in terms of quality improvement. However, it is noted that test scenario 3 outperformed the quality performance recorded in test scenario 2 with a variation of $3.25 \mathrm{~dB}$ gain. This significant performance is achieved within equal limited resource network constraints. However, the improvement in the quality performance is a result of systematic adaptation of the NALU base on the sensitivity of the media packets. Figure 3 presents the assessment of test results carried out with Akiyo test media sequence.

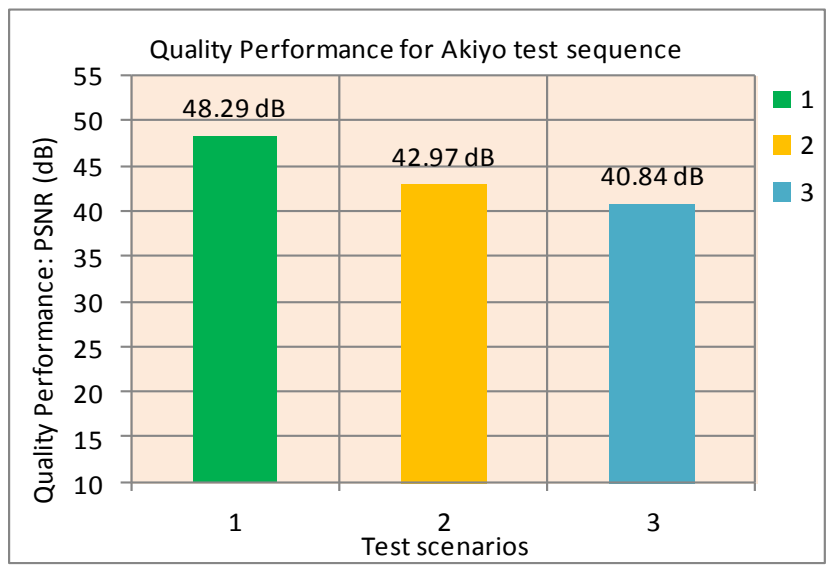

Figure 3: Quality Performance for Akiyo test sequence

Comparing the results obtained under three different test conditions. It has been observed that media quality performance obtained under scenario-1 outperforms that of test scenario- 2 and scenario-3 respectively. This is because the test scenario 1 was performed under error free channel conditions. Further observation from Figure 3, shows that test scenario 2 performs better compared to test scenario 3 with Akiyo test media sequence. Based on the observations from Figure 3 , at the same source bitrates, media quality performance at scenario 2 shows significant improvement compared to the quality performance scenario 3 . Thus, media packets with high sensitivity to channel errors 
transmitted through the wireless channel were delivered without much distortion much corrupted packets. It has also been observed that the quality performance of Akiyo test sequence with low priority performed better at the three test conditions. This is due to the fact the low sensitive media streams to channel errors experience negligible distortion at the network level. However, it has been observed that media distribution using the proposed technique improves the overall received quality performance of the tested media streams. Thus, the technique is capable of improving the quality of mobile multimedia communication services.

\section{Conclusion and Future Work}

Advanced technique for improved mobile multimedia communication services is discussed. The paper investigated the existing technologies for multimedia communication and proposed a technique capable of improving the quality of multimedia communication services over mobile network. The advance technique systematically adapt the NALU of the media packets based on the sensitivity of the media content. The contents with high sensitivity to channel errors are packetized uniquely compared to the media packets of low sensitivity to channel errors. The proposed advance technique saves the limited wireless network resources through intelligent adaptation of the NALU based on media stream error sensitivity. Test results recorded improvement in the overall received media quality performance compared to the conventional approach. Future work investigates further more advanced techniques to improve the quality performance of multimedia communication services.

\section{ACKNOWLEDGMENTS}

The authors are grateful to the participants who contributed to the successful conclusion of the research work. The contribution of Akwa Ibom State University to the research work is also acknowledged. However, the role of the publisher in reviewing process is appreciated. Thank you for the support.

\section{REFERENCES}

[1] S. Kumar, L. Xu, M.K. Mandal and S. Panchanathan, "Error Resiliency Schemes in H.264/AVC Standard" Journal of Visual and Image Representation, Elsevier, August 2005.

[2] A. J. Goldsmith and S. G. Chua, "Adaptive coded modulation for fading channels," Communications, IEEE Transactions on, vol. 46, pp. 595-602, 1998.

[3] W. T. Webb, "The modulation scheme for future mobile radio communications," Electronics and Communication Engineering Journal, pp. 167-176, August 1992.

[4] S. Sampei, "Rayleigh Fading Compensation for QAM in Land Mobile Radio Communications," IEEE Transactions on Veh. Tech., vol. 42, pp. 137-147, 1993.

[5] T. Wiegand, G. J. Sullivan, G. Bjontegaard, and A. Luthra, "Overview of the H.264/AVC video coding standard," IEEE Transactions on Circuits and Systems for Video Technology, vol. 13, pp. 560-576, 2003.

[6] A.H.Sadka, "Compressed Video Communications," John Wiley \& Sons, Limited, England, 2002. 
[7] I. E. G. Richardson, "H.264 and MPEG-4 Video Compression," John Wiley and Sons Limited. West Sussex, England, 20032003.

[8] G. Nur, S. Dogan, H. Kodikara Arachchi and A.M. Kondoz, "Impact of Depth Map Spatial Resolution on 3D Video Quality and Depth Perception", Processings of the $4^{\text {th }}$ IEEE 3DTV Conference, Tampere, Finland, 7-9 June 2010.

[9] D. Fleet and Y. Wiess, "Optical Flow Estimation" Handbook of Mathematical Models in Computer Vision, Springer, 2006.

[10] ITU-T and ISO/IEC, "H.264/AVC JM reference Software, 2004.

[11] L. Hanzo, P. Cherriman, and J. Streit, "Wireless Video Communications," Second Edition, IEEE Press, New York, United States of America, 2001.

[12] M. Vranjes, S. Rimac-Drlje, and K. Grgic, "Locally averaged PSNR as a simple objective Video Quality Metric," ELMAR, 2008. 50th International Symposium, pp. 17-20.

[13] ROHDE and SCHWARZ, "Mobile WiMAX MIMO Multipath Performance Measurements," WiMAX Forum, 2010.

[14] M. Wittmann, J. Marti, and T. Kurner, "Impact of the power delay profile shape on the bit error rate in mobile radio systems," IEEE Transactions on Vehicular Technology, vol. 46, pp. 329-339, 1997. 